\title{
Sistema de control de calidad para la automatización de la inspección de bebidas en plantas embotelladoras de Potosí, Bolivia
}

\author{
Quality control system for the automation of beverage inspection in bottling plants in Potosí, \\ Bolivia
}

Sistema de controle de qualidade para a automação da inspeção de bebidas em fábricas de engarrafamento em Potosí, Bolívia

\section{Sandro Rimberto Calcina Salvatierra \\ rimber11@gmail.com \\ ORCID 0000-0002-2494-5979}

Universidad Privada Domingo Savio, Potosí-Bolivia

Artículo recibido enero 2021 | Arbitrado en febrero 2021 | Publicado en mayo 2021

\section{RESUMEN}

En el estudio se desarrolló un sistema de control de calidad para la automatización de las plantas embotelladoras. Se parte de un diagnóstico de necesidades con base en la información de cinco gerentes y 15 trabajadores de cinco plantas embotelladoras de la ciudad de Potosí, Bolivia: EMBOSUR, PROVEL LTDA, CASCADA POTOSÍ, LIPEÑA. El objeto de estudio fue el control de la calidad, por su naturaleza se clasifica como una investigación proyectiva tecnológica, bajo el diseño de campo. Las técnicas de recolección de datos usadas fueron la observación y la entrevista; los instrumentos fueron la guía de observación y el guion de entrevista. Como resultado se presenta el sistema de control de calidad para la automatización el cual se construyó aplicando la Metodología Ágil XP, el lenguaje de programación $\mathrm{C}++$ y OpenCV para el procesamiento de imágenes y la teoría correspondiente para el diseño del Hardware para dar respuesta al diagnóstico de necesidades.

Palabras clave: Sistema de control de calidad, Inspección automatizada, embotelladoras en Potosí, Metodología Ágil $\mathrm{XP}$, programación $\mathrm{C}++$, OpenCV
ABSTRACT

RESUMO
The study developed a quality control system for the automation of bottling plants. It starts from a needs assessment based on information from five managers and 15 workers from five bottling plants in the city of Potosí, Bolivia: EMBOSUR, PROVEL LTDA, CASCADA POTOSÍ, LIPEÑA. The object of study was quality control, by its nature it is classified as a technological projective research, under the field design. The data collection techniques used were observation and interview; the instruments were the observation guide and the interview script. As a result, the quality control system for automation is presented, which was built by applying the Agile XP Methodology, the C ++ and OpenCV programming language for image processing and the corresponding theory for the design of Hardware to respond to the diagnosis of needs.

Key words: Quality control system, Automated inspection, bottling plants in Potosí, Agile XP Methodology, C ++ programming, OpenCV
O estudo desenvolveu um sistema de controle de qualidade para a automação de fábricas de engarrafamento. É baseado em uma avaliação de necessidades com base em informações de cinco gerentes e 15 trabalhadores de cinco fábricas de engarrafamento na cidade de Potosí, Bolívia: EMBOSUR, PROVEL LTDA, CASCADA POTOSÍ, LIPEÑA. O objeto de estudo foi o controle de qualidade, por sua natureza é classificado como uma pesquisa projetiva tecnológica, sob o conceito de campo. As técnicas de coleta de dados utilizadas foram observação e entrevista; os instrumentos foram o roteiro de observação e o roteiro de entrevista. Como resultado, é apresentado o sistema de controle de qualidade para automação, o qual foi construído aplicando a Metodologia Agile XP, a linguagem de programação $\mathrm{C}++$ e OpenCV para processamento de imagens e a correspondente teoria para o design de Hardware para responder ao diagnóstico de necessidades.

Palavras-chave: Sistema de controle de qualidade; Inspeção automatizada; engarrafadores em Potosí; Metodologia Agile XP Programação C ++; OpenCV 
INTRODUCCIÓN

Las empresas embotelladoras de bebidas deben generar al mercado productos de calidad bajo un estricto control financiero y regulatorio, para lo cual deben conseguir y mantener estándares internacionales como el cumplimiento de la Normas ISO 9000.

Un sistema de control de calidad automatizado para la inspección de bebidas ayuda a mantener la calidad del proceso y del producto, este sistema automatizado surge al fusionar múltiples especialidades como la informática, visión artificial y automatización. Las oportunidades para la implementación de estos sistemas se encuentran en tareas de inspección, selección de productos, control de calidad, embalaje y monitorización de procesos.

La automatización es la prioridad de muchas empresas, ya que permite el aumento de producción, el uso eficiente de las máquinas y de la materia prima a utilizar. Para estos sistemas actualmente se hace uso de la visión artificial, la cual, por medio de cámaras y software especializado, puede manejar procesos y verificar que el sistema funcione de manera óptima y acorde a los parámetros requeridos por los estándares de calidad y las exigencias del consumidor.

Son múltiples las ventajas que se pueden obtener al reemplazar los métodos tradicionales por sistemas de control de calidad automatizados. Estos sistemas brindan la posibilidad de inspeccionar toda la producción a grandes velocidades; además, permiten complementar los sentidos humanos como la vista y el tacto en aquellos lugares en donde la monotonía, velocidad, peligrosidad o el coste, entre otros, hacen inviable el uso de operarios, entre estos se encuentran los sistemas de control automatizados, los cuales pueden optimizar el desempeño durante los procesos de elaboración del producto; y permiten mejorar las actividades realizadas manualmente, mejora el rendimiento para una mayor rentabilidad, reducir riesgos, reducir desperdicio al controlar y ajustar el contenido neto y agilizar la elaboración de informes con la recolección de datos confiables.

El uso de la visión artificial en los sistemas automatizados muestra gran versatilidad, precisión, repetitividad, consistencia y reducción de costes. Así como también, las cámaras permiten la inspección en cada una de las piezas en vez de muestreos por lotes, y cada pieza es consistente al nivel de calidad de cada una. Así mismo, la visión artificial ayuda a acelerar el proceso de verificación y revisión para diferentes pruebas de calidad que se realiza antes de la salida del producto.

Las empresas embotelladoras en la ciudad de Potosí en Bolivia, en su mayoría, realizan inspecciones con operarios que revisan el llenado y la presencia de sustancias extrañas de manera visual sobre las botellas. La inspección o detección por operarios puede ocasionar errores por cansancio y desgaste visual, esto redunda en la aparición de errores en el producto embotellado. Estas empresas no se han consolidado completamente en el mercado financiero y aunque conocen los métodos de automatización no han invertido para su compra e instalación.

Las empresas embotelladoras al contratar operarios para el control de calidad producen el incremento en los costes de producción debido a la ejecución de tareas adicionales de clasificación y costes por reproceso. 
Esta investigación busca crear un sistema de visión artificial, que ayude a evaluar las bebidas embotelladas de una empresa. Este proceso se llevará a cabo en el área de control de calidad, detectando alguna impureza en las bebidas embotelladas y haciendo la revisión de nivel de volumen necesario mediante parámetros preestablecidos.

El problema de investigación se plantea: ¿Cuáles son las características de un sistema de control de calidad para la automatización de la inspección de bebidas en las plantas embotelladoras de la ciudad de Potosí, Bolivia?

Asimismo el objetivo que guía la investigación es desarrollar un sistema de control de calidad para la automatización de la inspección de bebidas en las plantas embotelladoras de la ciudad de Potosí, Bolivia.

\section{MÉTODO}

Se trató de una investigación proyectiva de tipo tecnológica ya que se generó una propuesta de un sistema control de calidad para la automatización de la inspección de bebidas en plantas embotelladoras con base en los resultados del diagnóstico de necesidades. La población estuvo constituida por cinco gerentes, encargados e ingenieros de producción y todos los trabajadores de las distintas áreas de cuatro empresas embotelladoras de la ciudad de Potosí, las cuales son: EMBOSUR, PROVEL LTDA, CASCADA POTOSÍ, LIPEÑA, para un total de 20 personas. Las técnicas utilizadas para la recolección de los datos fueron la entrevista y la observación. Se diseñaron dos guiones de entrevista, uno para los gerentes y encargados de las empresas para conocer los instrumentos o máquinas que utilizaban para el control de calidad, este guion constó de 8 preguntas no estructuradas. El otro guion de diseñó para conocer la información desde el punto de vista de los operarios de las plantas con la finalidad de diagnosticar los procesos que desarrollan en el control de calidad del llenado de botellas y la detección de alguna impureza en las mismas. El instrumento de observación fue una guía con 6 ítems, que permitió recoger datos sobre los procedimientos en cuanto al control de calidad en la inspección de volumen correcto y la detección de alguna impureza en las bebidas embotelladas. El diseño de investigación fue de campo ya que se recolectaron los datos en las plantas embotelladoras. La variable de la investigación se resume en el Cuadro 1. 
Cuadro 1. Variables de investigación

\begin{tabular}{|c|c|c|c|}
\hline Variables & Definición conceptual & Dimensiones & Indicadores \\
\hline $\begin{array}{l}\text { Inspección } \\
\text { de bebidas }\end{array}$ & $\begin{array}{l}\text { Sirven para examinar y medir las } \\
\text { características de un producto, así como } \\
\text { los componentes y materiales de los } \\
\text { cuales está elaborado, o de un servicio o } \\
\text { proceso determinado, todo ello utilizando } \\
\text { instrumentos de medición, patrones de } \\
\text { comparación o equipos de pruebas y } \\
\text { ensayos, todo con el fin de verificar si } \\
\text { cumple o no con los requisitos } \\
\text { especificados. }\end{array}$ & $\begin{array}{l}\text { Guía de } \\
\text { inspección }\end{array}$ & $\begin{array}{l}\text { Inspección de } \\
\text { volumen } \\
\text { Inspección de } \\
\text { impurezas }\end{array}$ \\
\hline
\end{tabular}

\section{RESULTADOS}

\section{Entrevista a los gerentes o encargados de las empresas embotelladoras}

El $80 \%$ de los gerentes y encargados entrevistados afirman que sus empresas no tienen un sistema de control de calidad automático, esto debido a que su empresa está aún en formación, pero si está en sus planes a un futuro adquirir máquinas automáticas que realicen dicho trabajo en el área de producción. Coincidieron que todo el personal de la empresa está comprometido con la calidad de sus productos, con el objetivo de posicionarse de su marca y la buena imagen de sus empresas con sus proveedores y clientes consumidores.

Los gerentes de las empresas que no tenían sistema de control de calidad indican que se realizaba mediante operarios que se encargan de dicha función utilizando la observación para la inspección de productos.

Las empresas embotelladoras no tienen equipos del todo automatizados en el área de control de calidad ya que usan personal para poder verificar que una botella no tenga impurezas usando linternas o reflectores para detectar algún objeto extraño dentro las botellas.

La mayoría de los gerentes y encargados entrevistados indican que la manifestación más clara de que la calidad, está cumpliendo en su empresa, sería que sus productos se van abriendo mercado y el aumento de la demanda en sus consumidores.

En referencia a que si su empresa verifica en forma periódica el grado de satisfacción que sus clientes obtienen con los productos que se les ofrece, los entrevistados respondieron que no cuentan con personal en esa área para poder realizar dicho trabajo debido a que recién se van formando en el ámbito empresarial.

Los entrevistados indican de que buscan evitar el más mínimo error posible ya que si cometieran una mala inspección al producto final corren el riesgo de tener una mala imagen de la empresa y por ende la pérdida de clientes, para poder evitar dichos errores los encargados de producción están capacitando constantemente sobre el control de calidad a todos los empleados de la empresa. 
También declararon que tienen como objetivo tener un producto de calidad en el mercado para ganar la confianza de los consumidores y con el tiempo buscar el reconocimiento de sus marcas a nivel nacional.

Entrevista a los empleados que trabajan en las empresas embotelladoras.

Los trabajadores de las distintas empresas con respecto a la pregunta de que si hay un sistema de control de calidad respondieron indicando que no hay un sistema de inspección de bebidas embotelladas, algunos trabajadores indicaron que sí, pero no del todo automatizado ya que en algunas áreas se utilizan personal para que realice de alguna manera el control de calidad.

Indican que están comprometidos con la calidad ya que la empresa les va capacitando constantemente en las actividades que les son encomendadas en la línea de producción, distribución y ventas, lo que redunda en la obtención de beneficios constantes para ellos, sus familias y los socios.

Para el control de alguna impureza en el contenido de las botellas los empleados entrevistados indicaron que se les realiza mediante personal que trabaja en el área utilizando linternas o reflectores para poder detectar alguna impureza dentro de la botella, observando muy atentamente a cada botella que pasa por la iluminación.

Las herramientas se utilizan para medir el nivel correcto de llenado de botellas, los trabajadores indicaron que se realiza automáticamente mediante máquinas llenadoras que se encargan del llenado correcto de las botellas, eso de las empresas que cuentan con dicha maquina llenadora, mientras los trabajadores de las empresas que no tienen la maquina llenadora indicaron que utilizan una muestra para poder llenar a las demás botellas, utilizando operarios que realicen dicho trabajo.

Los empleados coinciden con la mayoría de los Gerentes y encargados entrevistados quienes indicaron que la manifestación más clara que la calidad se está cumpliendo en su empresa seria que sus productos se van abriendo mercado argumentando de que cada vez los distribuidores piden más productos y por ende sus labores dentro de las empresas cada vez es más arduo.

Los entrevistados en su mayoría respondieron que desconocen cómo la empresa verifica el grado de satisfacción del cliente, consideran que un aumento en las ventas podría ser un indicador de satisfacción.

Coinciden en evitar cometer errores en la inspección de los productos por el riesgo de perder la confianza de los consumidores, señalan que reciben constantes capacitaciones con los encargados de producción de la empresa para asegurar la calidad del producto.

Las empresas embotelladoras contribuyen a evitar que se cometan errores ya que es una herramienta de autoevaluación y de autocontrol que les permiten monitorear en tiempo real el que hacer de la empresa y por otro lado, comparar los resultados alcanzados en relación con lo programado y así determinar las posibles desviaciones, a fin de promover acciones correctivas necesarias para el cumplimiento de los planes y facilitar alcanzar las metas previamente establecidas.

Coinciden en opinar que un sistema de control de calidad automatizado les daría mayor seguridad que el producto final cumple con los criterios de calidad. 
De los resultados de la observación cabe destacar que las empresas embotelladoras que tienen sistemas para el control de volumen lo realizan utilizando llenadoras ponderales que consiste en pesar el producto que entra en la botella tras haber calibrado el sensor con la tarea correspondiente las que no cuentan con este tipo de sistemas de control de volumen utilizan operarios que se encargan de llenar las botellas basándose en una muestra que se les da para que les sirva como guía en el llenado.

Con respecto al control de impurezas, todas las empresas embotelladoras lo realizan mediante operarios que están atentos al paso de las botellas por la banda transportadora, utilizando reflectores o linternas para verificar si hay o no impurezas en el contenido de las botellas, las empresas que no cuentan con banda transportadora inspeccionan una por una antes de ser empaquetado.

El control del nivel de llenado lo realizan basándose como guía en una botella de muestra, ocupando operarios que realizan la tarea mediante el sentido de la vista para verificar el nivel de llenado correcto, este método no es eficiente ya que el ser humano puede ir desgastándose físicamente e ir cometiendo errores de verificación por el cansancio.

La inspección o detección de impurezas dentro de las botellas lo realizan mediante operarios, quienes usando linternas y el sentido de la vista, los operarios revisan cada una de las botellas que pasa por la banda transportadora buscando residuos o sustancias extrañas al contenido. Este método no siempre da buenos resultados, ya que al trabajar tiempos completos, la luz puede ir dañando a los ojos del trabajador, causar el agotamiento físico y poner en riesgo de que el operario no cumpla con su trabajo de manera eficiente.

\section{Propuesta}

Considerando los resultados del diagnóstico se plantea la propuesta del sistema de control de calidad, que permita realizar la inspección de bebidas embotelladas tanto en el control de nivel de llenado como también en la detección de alguna impureza. Este sistema facilita el proceso de control de calidad en beneficio del personal operativo del área de producción y de los consumidores.

La propuesta integra el software y el hardware, se desarrolló con el lenguaje de programación $\mathrm{C}++$ y OpenCV para el procesamiento de imágenes y consta de una banda transportadora encargada de generar el movimiento de las botellas a evaluar, hasta ser detectadas por un sensor infrarrojo, siendo controlada por una placa Arduino posicionada en paralelo con una cámara, que permite obtener las imágenes de las botellas para realizar el control de llenado y la detección de impurezas.

Para el diseño del software se utilizó el lenguaje de programación $\mathrm{C}++$ ya que permite la programación estructurada y la programación basada en objetos (Sánchez, 2004). También se hizo uso del Sqlite que es una biblioteca escrita en leguaje C que implementa un Sistema de gestión de bases de datos transaccionales SQL autocontenido, sin servidor y sin configuración. El código de SQLite es de dominio público y libre para cualquier uso, ya sea comercial o privado (Pressman, 2010). Se complementó con el uso de la multiplataforma OpenCV la cual contiene más de 500 funciones que abarcan una gran gama de áreas en el proceso de visión, como reconocimiento de objetos, calibración de cámaras, visión 
estéreo y visión robótica. Se eligió usar la biblioteca Opencv porque es una herramienta de visión artificial de código abierto que permite realizar el procesamiento de las imágenes (Furfaro, 2010).
Otro elemento para la construcción del software fueron los algoritmos. El algoritmo Grayscale (Guzmán Ramírez, Jiménez, Pérez y Pogrebnyak, (2011) para convertir las imágenes a escala de grises, se observa el efecto de la conversión en la Figura 1.

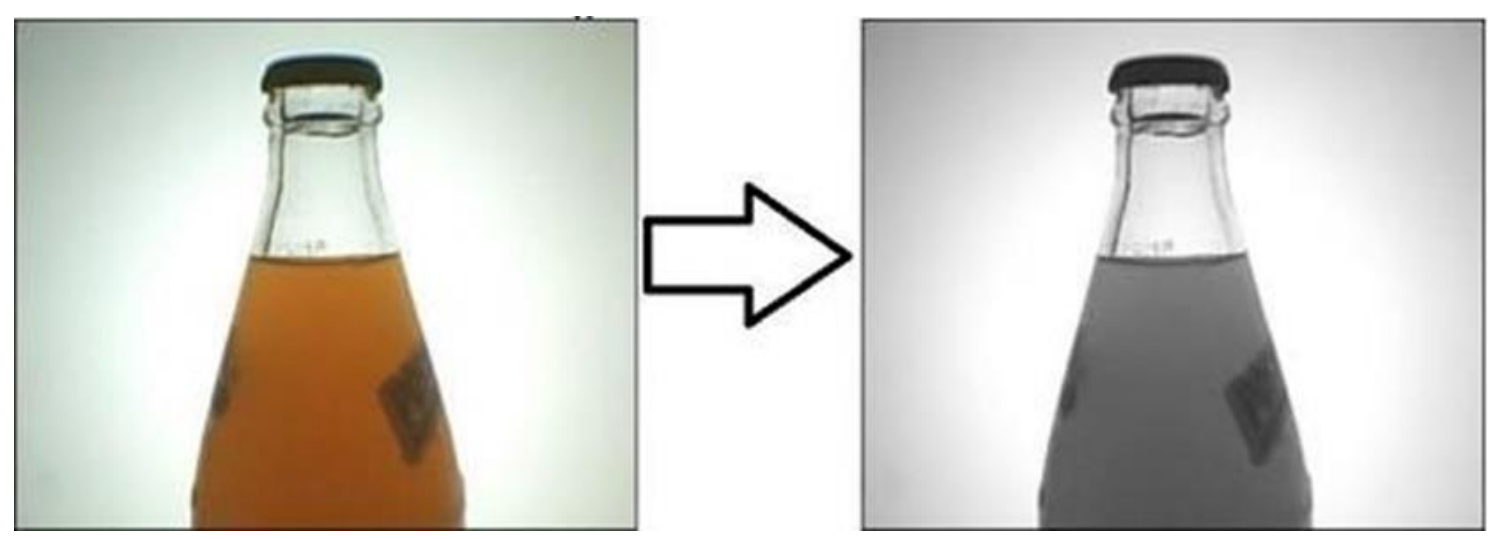

Figura 1. Conversión a escala de grises

Asimismo, el algoritmo de Canny (1986) se empleó para encontrar los bordes de las imágenes, e ir trabajando sobre ellas para detectar el nivel de llenado correcto del contenido de las botellas, el resultado del tratamiento de las imágenes se observa en la Figura 2.

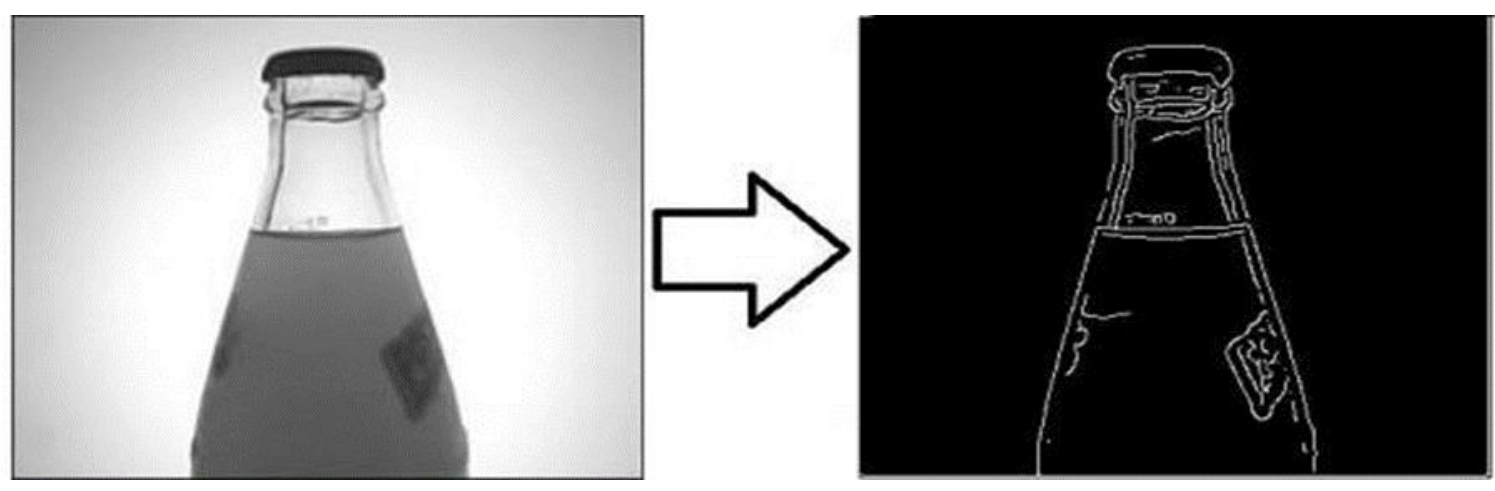

Figura 2. Conversión a borde de imagen aplicando algoritmo de Canny.

Otro algoritmo fue el HSV el cual se utilizó para dar más intensidad a los bordes de las imágenes para trabajar con mayor comodidad en el procesamiento. El algoritmo de búsqueda o seguimiento de contornos el Contour Following Algorithm
(Courant, 1996) para la búsqueda de contornos en las imágenes procesadas. También se usó el Algoritmo de dibujo de contorno que permitió dibujar los contornos encontrados en las imágenes procesadas en tiempo real e indicar si se 
encontró algún residuo dentro de las botellas (Dougherty, 1992 y Pajares, 2001).

En este mismo orden de ideas el Hardware del sistema de control de calidad quedó conformado por: Arduino, Motor DC, Sensor de Reflexión infrarrojo Evasor de Obstáculos, Driver dual para motores (FullBridge) - L298N, Cámara Web, Webcam Full HD - Logitech C920 y Lámpara Led (Malvino y Bates, 2007).

Arduino es una plataforma de prototipos electrónica de código abierto (open-source) basada en hardware y software flexibles y fáciles de usar. Permite crear objetos o entornos interactivos. Arduino está basado en micro controladores ATMEGA8 y ATMEGA168 de Atmel. Se ha seleccionado este dispositivo para generar la comunicación con Visual $\mathrm{C}++$, que es el encargado de controlar el sistema. Es de bajo costo y de fácil comunicación con diferentes softwares. Posee puertos de salida y entrada para señales digitales, las cuales son usadas para generar las órdenes al sistema (Ver figura 3).

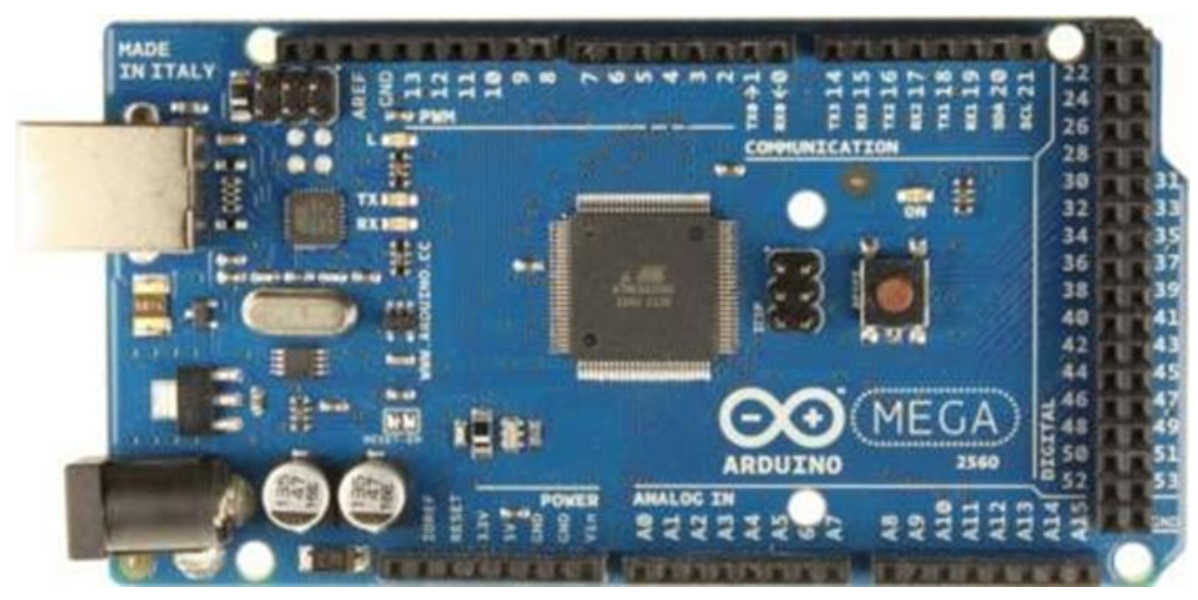

Figura 3. Arduino Mega 2560.

El Motor DC este dispositivo se eligió para poder mover la cinta transportadora que se realizó para simular el movimiento de las botellas. (Ver Figura 4).

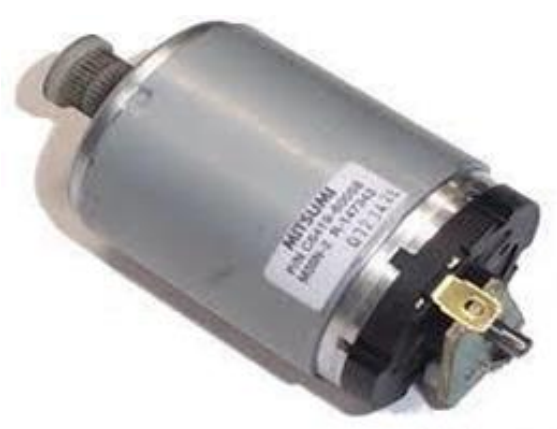

Figura 4. Motor de corriente continua. 
El Sensor de Reflexión infrarrojo Evasor de Obstáculos consiste en un detector de obstáculos infrarrojo es un dispositivo que detecta la presencia de un objeto mediante la reflexión que produce en la luz. Este sensor se eligió para poder detectar la presencia de las botellas mientras avanza la banda transportadora, enviando una señal de salida que indica detener la banda y capturar la imagen con la cámara. (Ver figura 5).

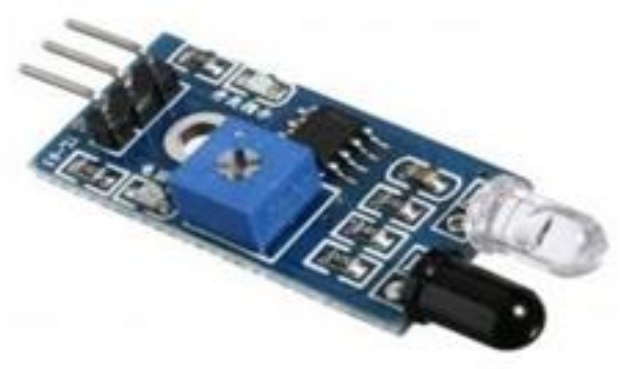

Figura 5. Sensor de Reflexión infrarrojo.

El Driver dual para motores (Full-Bridge) - L298N este dispositivo se usó para poder controlar el motor de la banda transportadora, recibiendo señales del sensor infrarrojo mediante la placa Arduino y así cumplir las órdenes de avanzar o detener el motor (Ver figura 6).

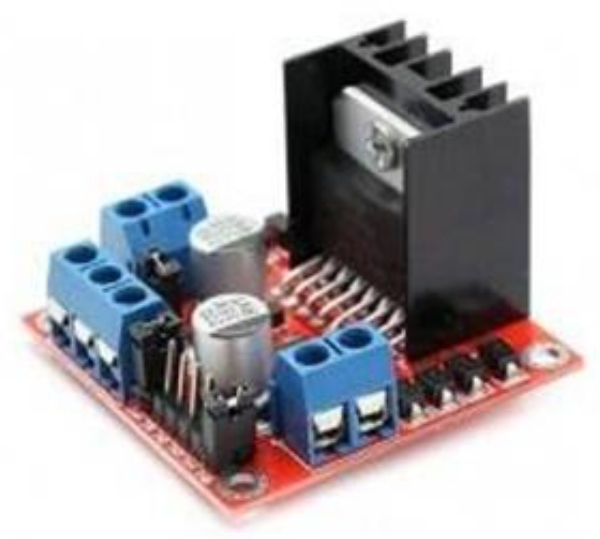

Figura 6. Módulo L298N.

La Cámara Web, Webcam Full HD - Logitech C920 en el desarrollo del sistema se utiliza la cámara web para poder capturar las imágenes y así poder procesarlas y la Lámpara Led permite resaltar la imagen de las botellas y así tener una mayor precisión en la inspección tanto de nivel y de impurezas (Ver figura 7). 

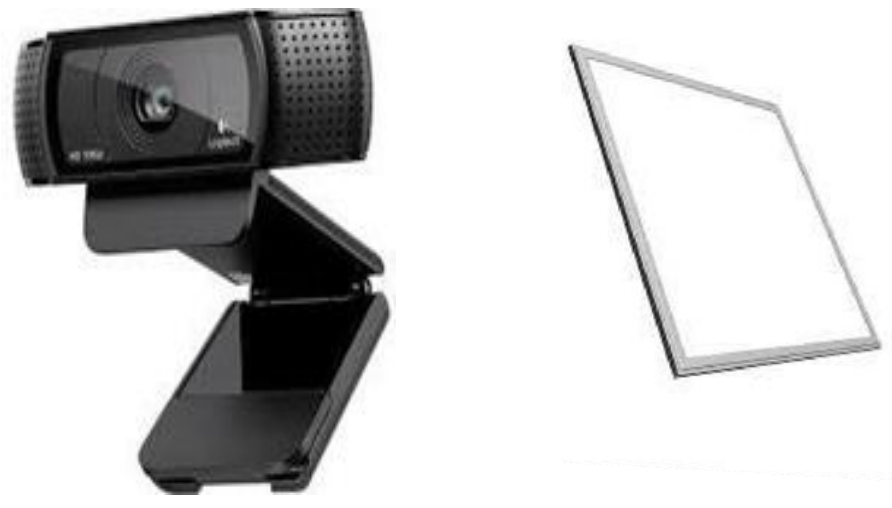

Figura 7. Cámara web y lámpara LED

Para la realización del proyecto se eligió la metodología ágil XP la cual consiste básicamente en ajustarse estrictamente a una serie de reglas que se centran en las necesidades del cliente para lograr un producto de buena calidad en poco tiempo, sus ventajas son que potencia las relaciones interpersonales como clave para el éxito del desarrollo de software, promueve el trabajo en equipo, preocupándose en todo momento del aprendizaje de los desarrolladores y estableciendo un buen clima de trabajo.

Las Herramientas de la Metodología XP son: Historias de Usuario según las recomendaciones de Letelier y Penades, (2006); Tareas de ingenierías (Task Card) según (Ferreira Escutia, 2013) Pruebas de aceptación, Según (Chiluisa Pallo y Loarte Cajamarca, 2014). Tarjetas CRC (Clase Responsabilidades -Colaboradores), Las Tarjetas CRC permiten conocer que clases componen el sistema y cuales interactúan entre sí. Se dividen en tres secciones: Nombre de la Clase, Responsabilidades y Colaboradores. (Chiluisa Pallo y Loarte Cajamarca, 2014). Los roles de la metodología XP son: programador, cliente, Encargado de pruebas (Tester), Encargado de seguimiento (Tracker), Entrenador (Coach), Consultor, Gestor (Big boss).

La Programación Extrema consta de cuatro fases, las cuales son: Planeación, diseño, codificación y pruebas.

El sistema de control de calidad está constituido por los módulos que resultaron del diagnóstico de necesidades, estos son: (a) Sesión, (b) Nivel de llenado, (c) Detección de impurezas, (d) Reportes y (e) Control de la banda transportadora.

Asimismo la información recolectada a través de "Las Historias del Usuario" para la inspección de bebidas embotelladas son: (a) Acceso al Sistema, (b) Gestión de Usuario, (c) Control del nivel de llenado de botellas, (d) Detección de impurezas en el contenido de las botellas, (e) Reportes, (f) Control con Arduino del motor y sensor infrarrojo en la banda transportadora. En función de estas Historias se definió el plan de entrega del proyecto del sistema de control.

Se diseñan los respectivos diagramas de: (a) clase, (b) casos de uso, (c) secuencia, (d) componentes, (e) despliegue (f) flujo del sistema, (g) circuitos electrónicos, en las Figuras 8 y 9 y 10 pueden observarse los diagramas de secuencia, componentes y despliegue respectivamente. 


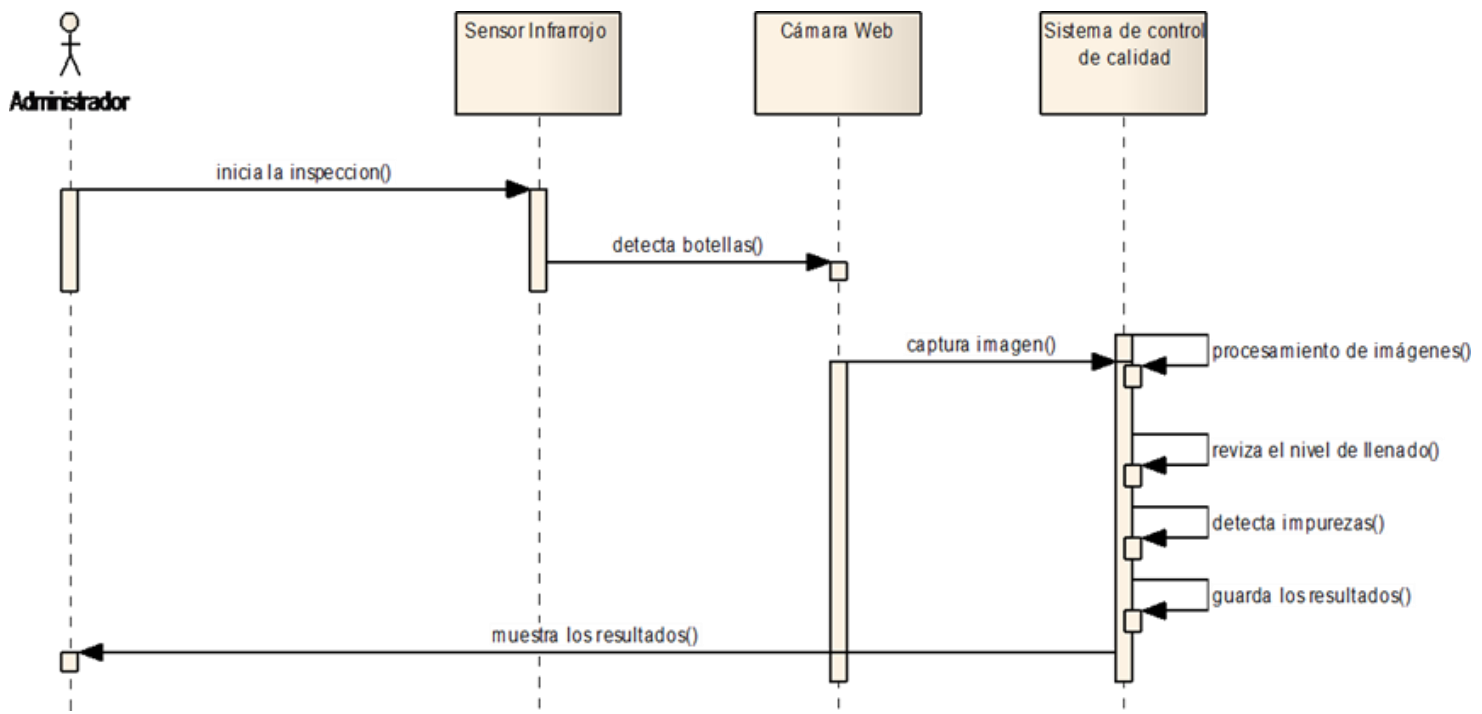

Figura 8. Diagrama de secuencia.

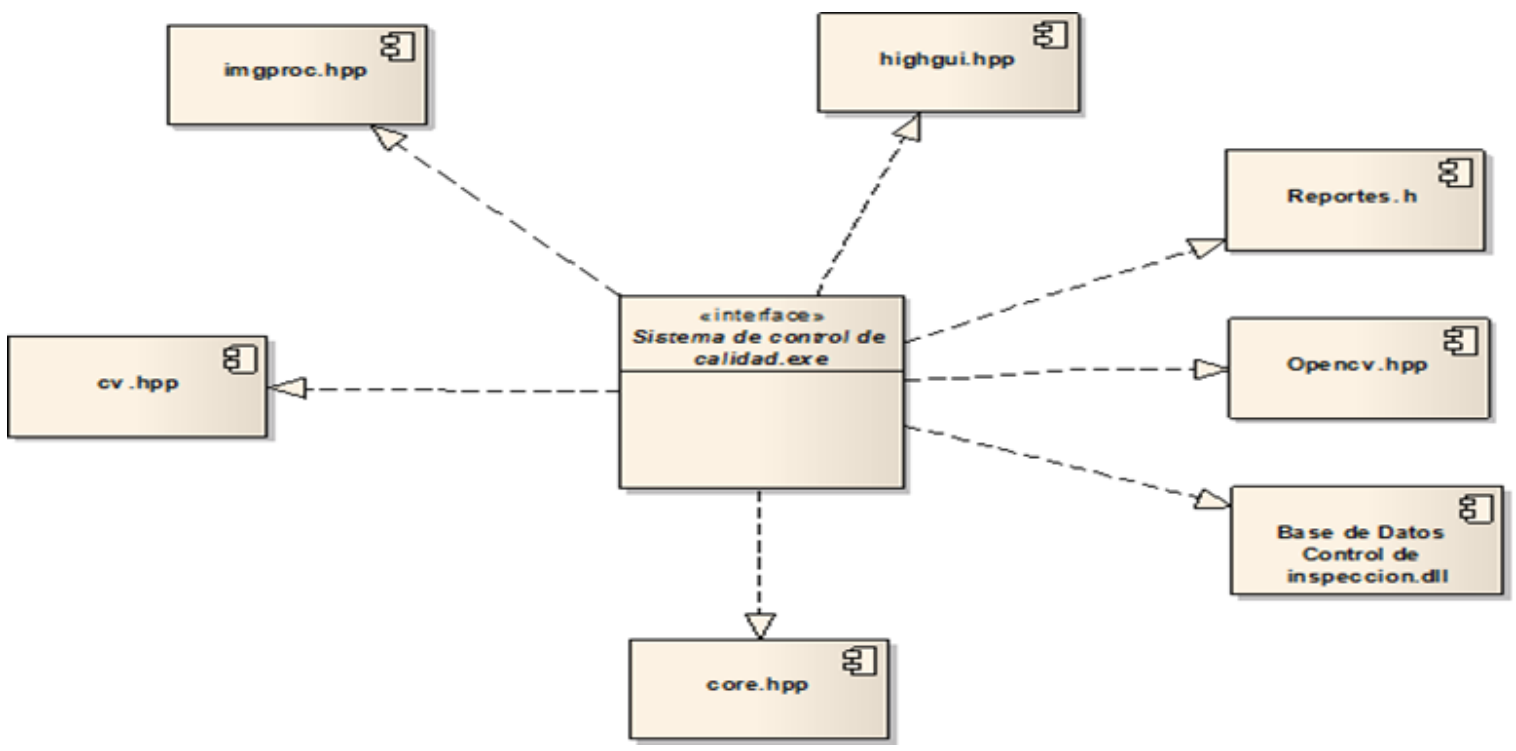

Figura 9. Diagrama de componentes. 


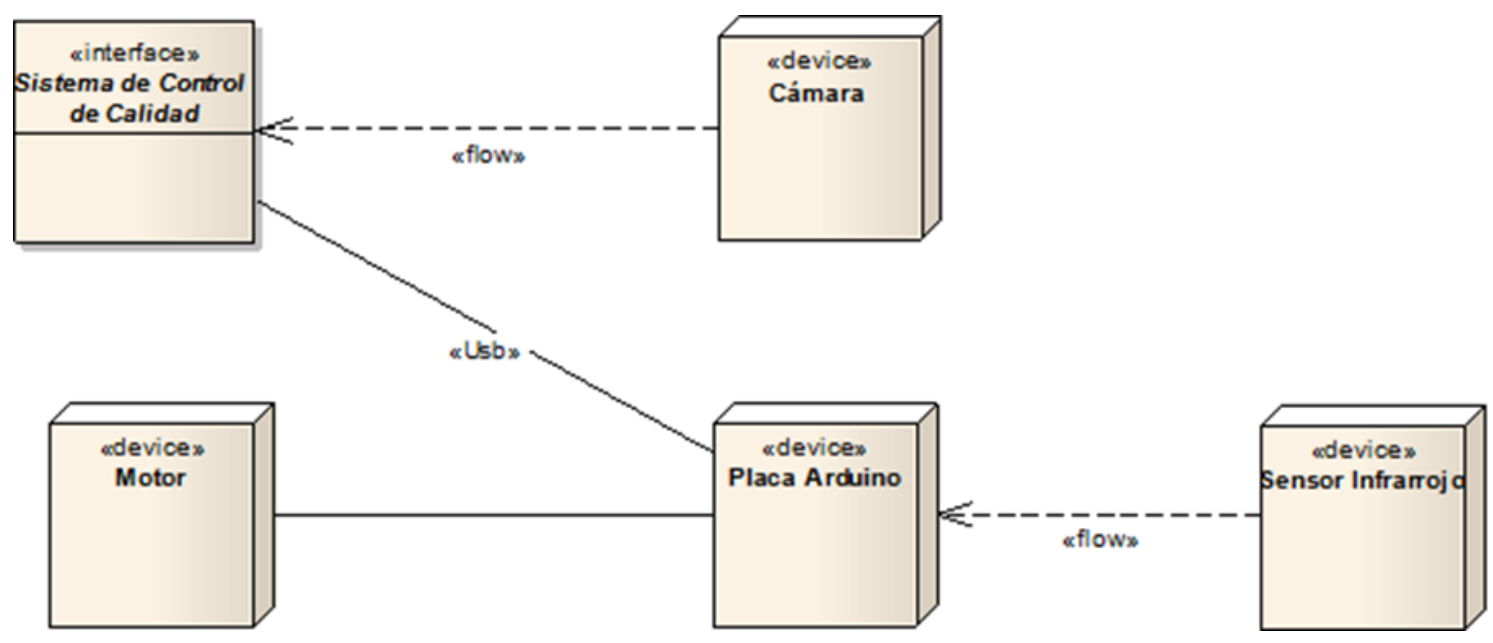

Figura 10. Diagrama del despliegue.

Aplicando las herramientas de la metodología de Programación Extrema XP, se realizan tres Iteraciones. Con la Primera Iteración se desarrolla el módulo de Sesión y el de Gestión de Usuario, se realizaron cinco tareas de ingeniería y dos pruebas de aceptación. En la figura 11 se muestra el resultado.

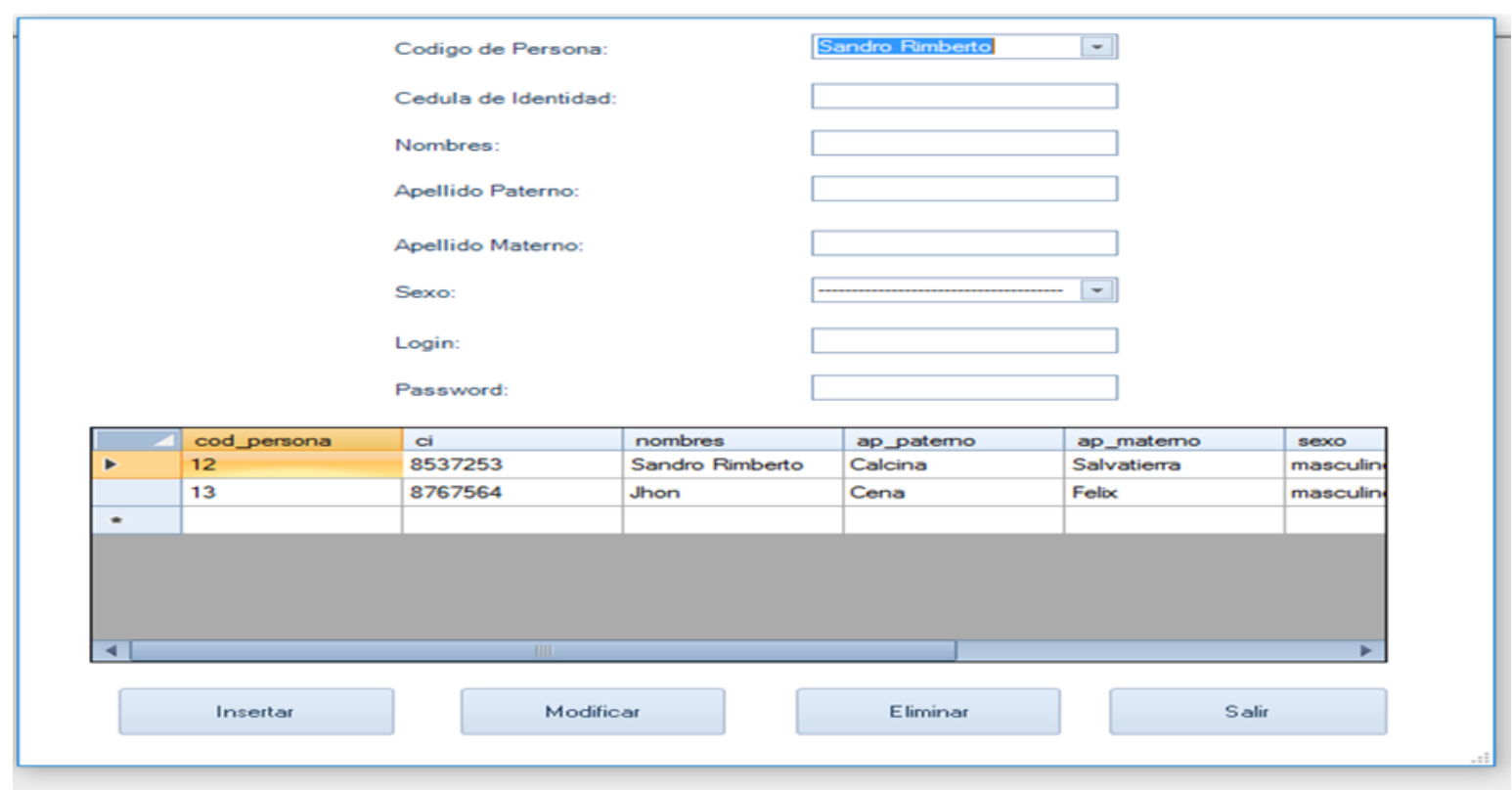

Figura 11. Caso de prueba: Gestión de Usuario

En la Segunda Iteración se desarrolla el módulo de Control del nivel de llenado de botellas. Se utilizaron 2 historias del usuario y 14 tareas de ingeniería y 2 pruebas de aceptación. En las figuras 12 y 13 se observa el resultado de los casos de prueba. 


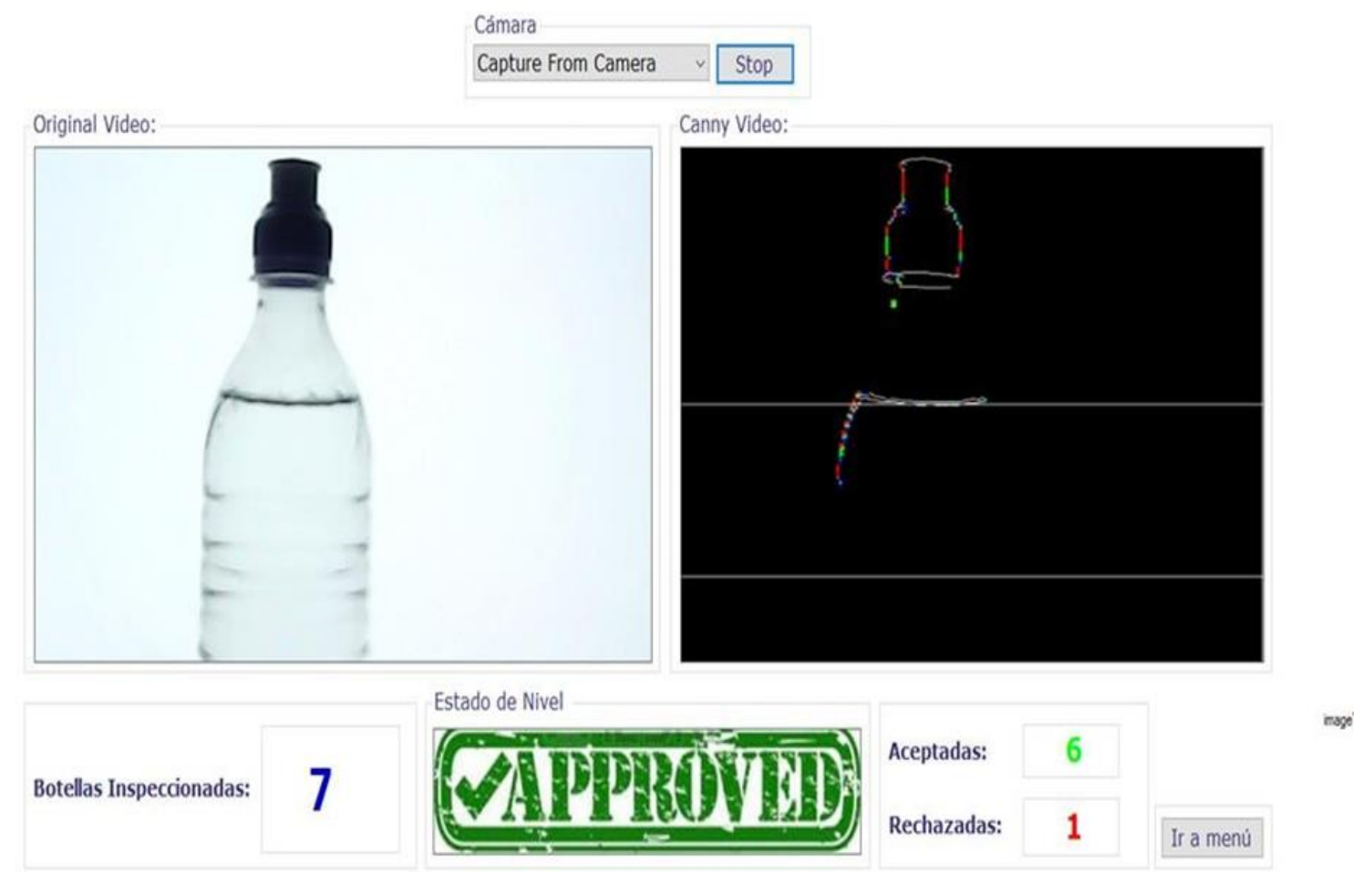

Figura 12. Caso de prueba: Control del nivel de llenado de botellas.

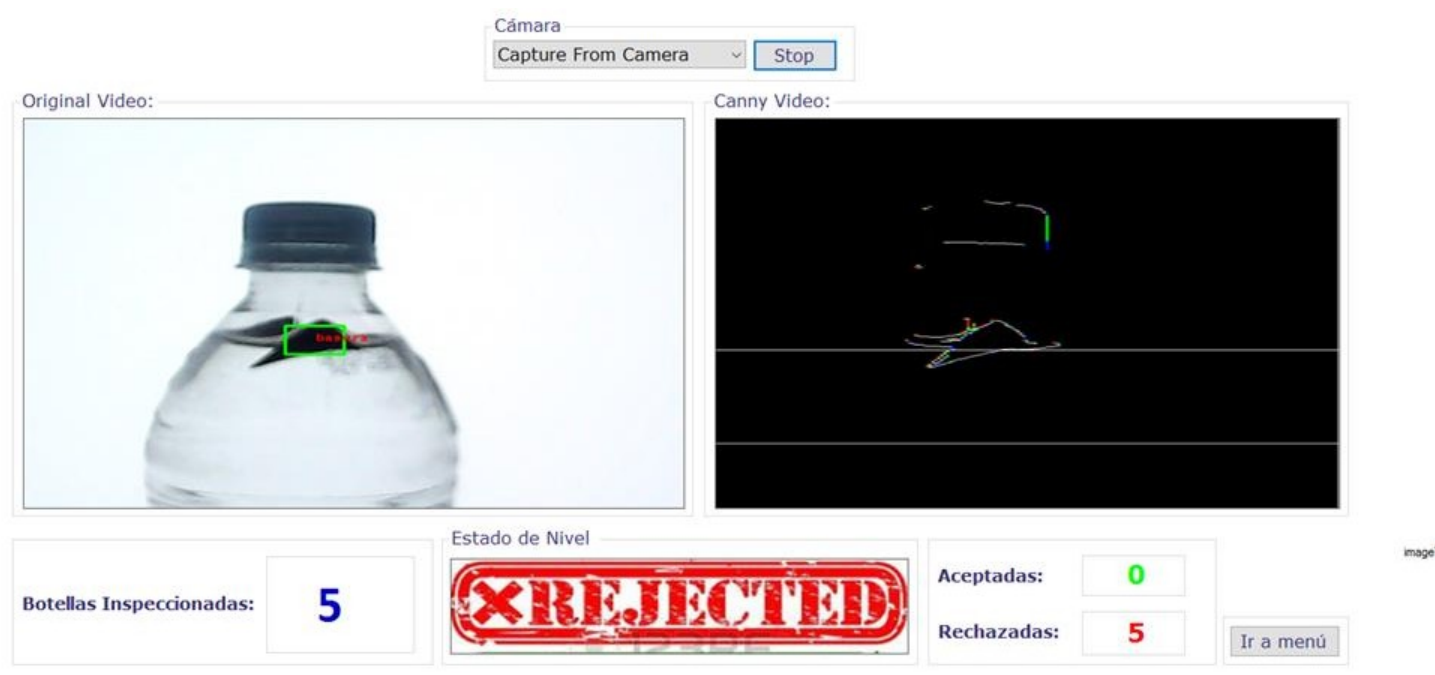

Figura 13. Caso de prueba: Detección de impurezas en el contenido de las botellas. 
Para la Tercera y última Iteración se desarrolló el módulo Reportes, el cual es generado a partir de la inspección de bebidas y el módulo de control de la banda transportadora. Para el diseño y la codificación se emplean las herramientas de la metodología XP. Se utilizaron dos historias de usuario, cinco tareas de ingeniería y dos pruebas de aceptación, los resultados pueden ser observados en las figuras 14 y 15.

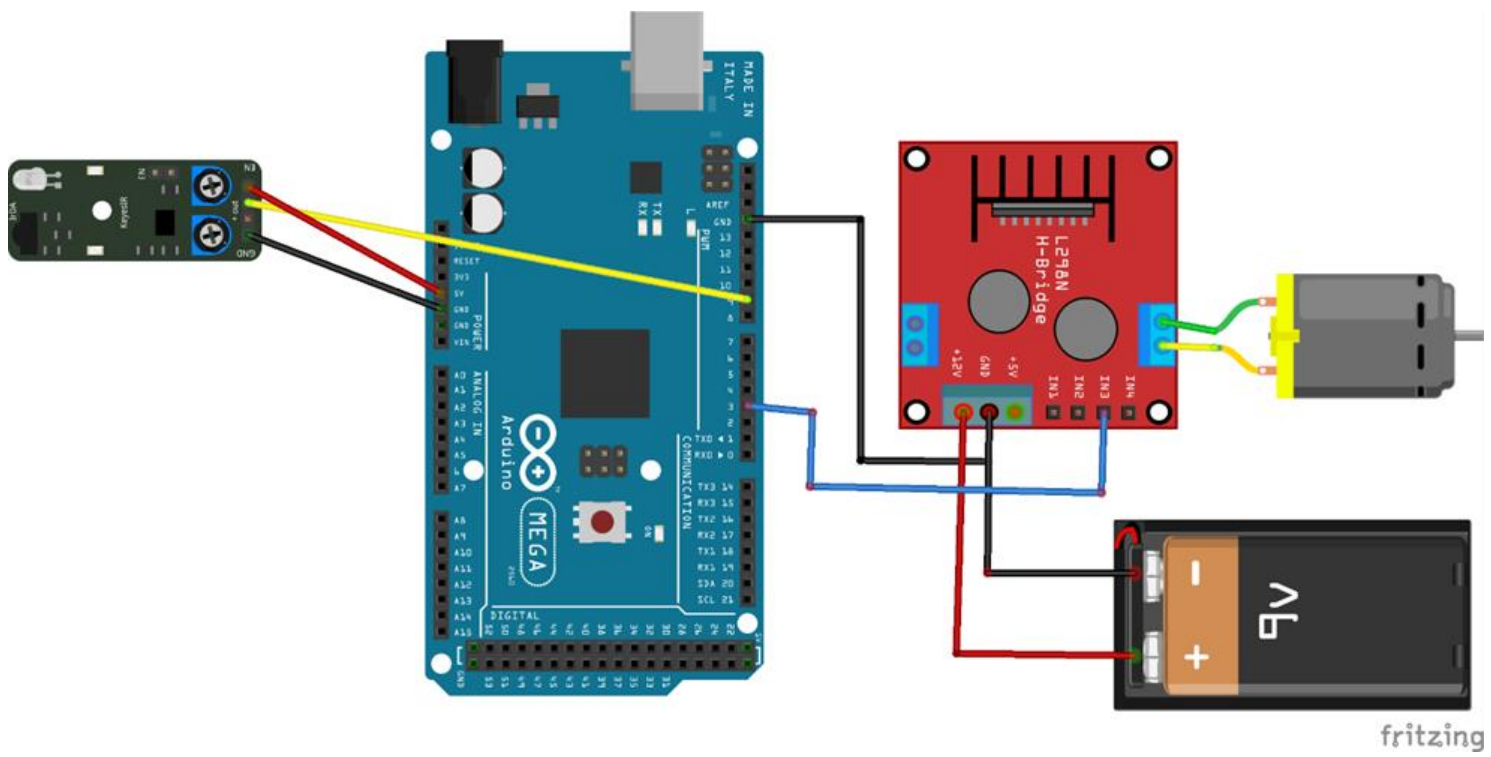

Figura 14. Caso de prueba: Reportes

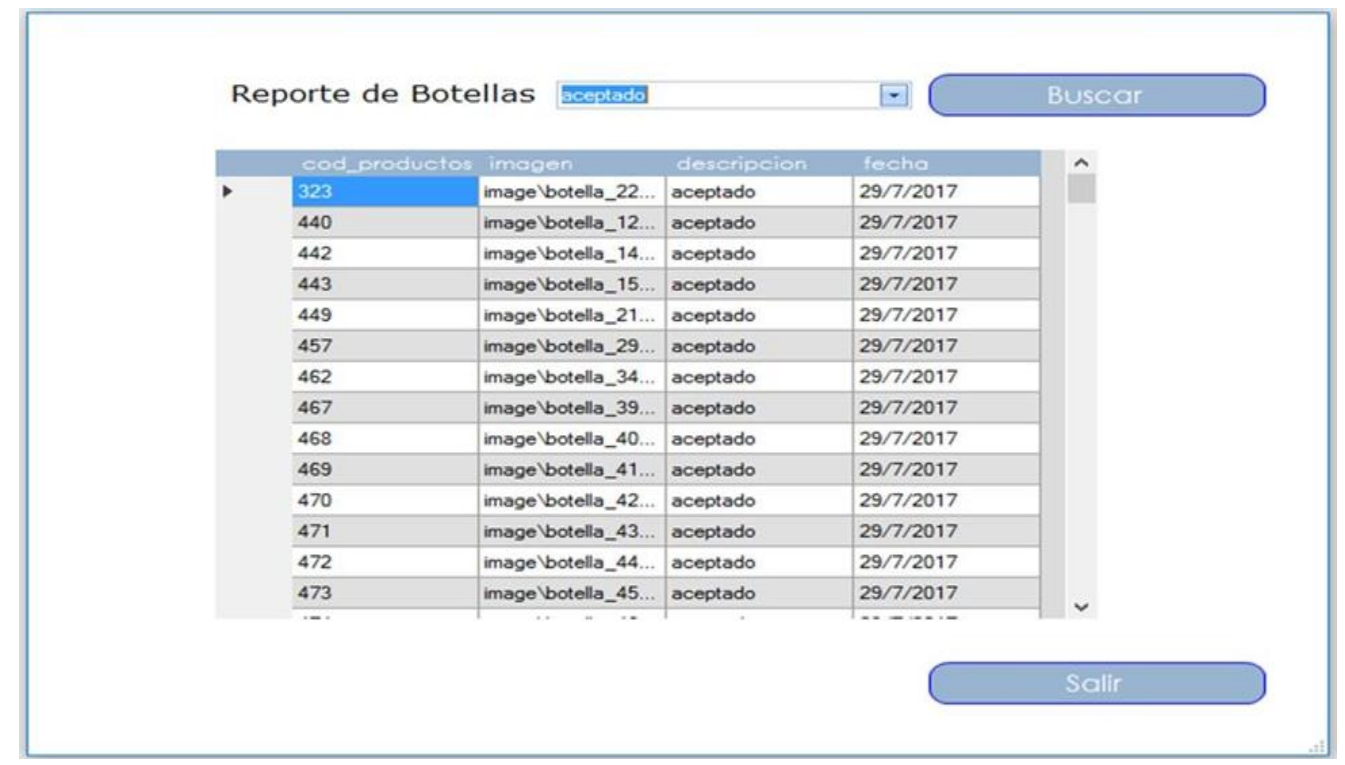

Figura 15. Caso de prueba: Control de banda transportadora. 


\section{CONCLUSIONES}

Las empresas embotelladoras de la ciudad de Potosí: EMBOSUR, PROVEL LTDA, CASCADA POTOSÍ, LIPEÑA realizan las actividades de control de calidad y la inspección de bebidas con operarios que se encargan de inspeccionar las botellas mediante observación visual, ya que no cuentan con sistemas automáticos de control de calidad. Esto puede ocasionar errores en el producto final y elevación de los costes de producción. Las empresas embotelladoras de este estudio tienen la necesidad de un sistema de control de calidad para la automatización de sus procesos de inspección de bebidas.

La propuesta de automatización denominada Sistema de Control de Calidad para las empresas embotelladoras se compone del software desarrollado a través del el lenguaje $\mathrm{C}++$, la biblioteca OpenCV y la metodología XP para su planificación y ejecución; el hardware se construyó con base en una placa Arduino. Esta propuesta se sometió a las pruebas y fue aceptada por los usuarios finales.

\section{REFERENCIAS}

Canny, A. (1986) Computational Approach to Edge Detection, IEEE Trans. on Pattern Analysis and Machine Intelligence, 8(6), 679-698

Chiluisa Pallo, A. y Loarte Cajamarca, B. (2014). Desarrollo e implementación del sistema de control de inventarios y gestión de laboratorios para la Facultad de Ciencias de la escuela
Politécnica Nacional, trabajo de grado. Universidad Politécnica Nacional: Quito, Ecuador. Disponible: https://bibdigital.epn.edu.ec/bitstrea m/15000/7732/1/CD-5638.pdf

Courant, R. (1996). ¿Qué es?: Una aproximación matemática elemental a ideas y métodos. Books google.com

Dougherty, E. R. (1992). Introduction to Morphological Image Processing. Bellingham: SPIE Optical Engineering Press

Ferreira Escutia, R. (2013). XP Extreme programing

Furfaro, A. (2010). Manejo de Bibliotecas OpenCV. Opencv org

Guzmán Ramírez, E., Jiménez, O., Pérez, A. y Pogrebnyak, 0. (2011). Grayscale Image Segmentation Based on Associative Memories. Computación y Sistemas, 15(2), 149-162. Disponible: http://www.scielo.org.mx/scielo.php?s cript=sci_arttext\&pid=S140555462011000400003\&lng=es\&tlng=en

Letelier, 0. y Penades, M. (2006). Metodologías agiles para el desarrollo del software, 5(26). Disponible: https://dialnet.unirioja.es/servlet/arti culo? codigo $=1983605$

Malvino, A. y Bates, D. (2007). Principios de electrónica (7aㅡ ed.). Madrid: Mc Graw Hill Interamericana de España

Pajares, G. (2001). Visión por Computador. Imágenes digitales y aplicaciones. Colombia: Ra-Ma

Pressman, 2010 Ingeniería del software. Enfoque práctico. España Mc Graw Hill Interamericana

Sánchez, J. (2004). C, Manual de referencia. Madrid: Osborne McGraw-Hill 